\title{
Estudio de Ruido en Aulas de Institución Educativa de Nivel Superior
}

\section{Noise Study in Classrooms of Higher Education Institution}

\author{
T. A. Arenas Ramos' ${ }^{1}$, K. G. Gómez Bull', M. M. Vargas Salgado ${ }^{1}$ \\ ${ }^{1}$ Universidad Autónoma de Ciudad Juárez
}

\begin{abstract}
RESUMEN
Este trabajo se realizó con el objetivo de evaluar el ruido existente en aulas de una escuela de nivel superior, mismas que tienen precedentes de alta percepción de ruido. La metodología bajo la que se trabajó fue de tipo descriptivo, cuantitativo, no experimental y transversal, y el desarrollo de la misma fue basado en la norma mexicana NOM-011-STPS-2001, para lo cual se recabaron 50 lecturas por muestra, en este caso en cuatro aulas, con cuatro puntos en cada una por prueba realizada. Una vez obtenidos los datos, se obtuvieron estadísticos descriptivos para determinar el cumplimiento con la norma de la Secretaría del Trabajo y Previsión Social (STPS). Como resultado se encontró que el promedio más alto de ruido fue el aula 4 con clase, que fue de $61.61 \mathrm{dBA}$, mientras que con salón vacío el promedio más bajo se encontró en el aula 2, con $39.50 \mathrm{dBA}$. En conclusión, es importante conocer la cantidad de decibeles a los que se encuentran expuestos alumnos y docentes, ya que al exceder de $80 \mathrm{~dB}$ por tiempo prolongado, estos pueden presentar problemas auditivos. Además, con este trabajo se determinó que los resultados obtenidos se encontraron dentro de los parámetros determinados por la norma y se estableció que el aula con mayor presencia de ruido fue la identificada como 4 , con $68.10 \mathrm{dBA}$.
\end{abstract}

PALABRAS CLAVE: Ruido; equipos de medición de ruido; evaluación de ruido.

\section{ABSTRACT}

This work was carried out with the objective of evaluating the existing noise in classrooms of a higher education institute, which have precedents of high perception of noise. The methodology used was descriptive, quantitative, non-experimental and transversal, the development of which was based on NOM011-STPS-2001. For which 50 readings were collected per sample, in this case there were four classrooms, with four points in each one per test performed. Once the data were obtained, descriptive statistics were acquired to determine compliance with the STPS standard. As a result, it was found that the highest noise average was class 4 , full classroom, that was $61.61 \mathrm{dBA}$, while with the lowest empty classroom it was found in class 2 with $39.50 \mathrm{dBA}$. In conclusion, it was determined that the results obtained were within the parameters determined by the norm and it was established that the classroom with the greatest presence of noise was identified as 4 with $68.10 \mathrm{dBA}$.

KEYWORDS: Noise; noise measurement equipment; noise evaluation.

\section{Correspondencia:}

DESTINATARIO: Karla Gabriela Gómez Bull INSTITUCIÓN: Universidad Autónoma de Ciudad Juárez DIRECCIÓN: Av. Plutarco Elías Calles 1210, C. P. 32410, Ciudad Juárez, Chihuahua, México.

CORREO ELECTRÓNICO: karla.gomez@uacj.mx

\section{Fecha de recepción:}

24 de julio de 2019

Fecha de aceptación:

11 de septiembre de 2019 


\section{INTRODUCCIÓN}

El ruido se define como aquellos sonidos con ciertos niveles de presión acústica que al ser combinados con un tiempo de exposición pueden ser nocivos a la salud de las persona ${ }^{[1,2]}$. Incluso se considera que puede tener efectos en la conducta social ${ }^{[3]}$. Este contaminante proviene de diferentes fuentes, entre las que se encuentran la tecnología, el desarrollo industrial e incluso las instituciones educativas, teniendo en común la intervención del ser humano ${ }^{[4]}$. El ruido ha adquirido cada vez mayor importancia y se ha incluido dentro de los factores ambientales que son causantes de daños nocivos sobre la salud física y mental de las personas ${ }^{[5]}$.

Este agente contaminante está presente en diversos contextos, tales como organizacional, laboral, de recreación, social e incluso en el educativo ${ }^{[6,7]}$. Particularmente en el último se da un ambiente poco óptimo para concentrarse y lograr un aprendizaje significativo, y es de resaltar que en las instituciones educativas tanto los maestros como los alumnos son más susceptibles a este factor de riesgo ${ }^{[8]}$. En la Tabla 1 se puede observar el impacto físico de acuerdo con la cantidad de decibeles a los que se encuentra expuesta una persona ${ }^{[9]}$.

TABLA 1.

IMPACTO FÍsICO DEL RUIDO

\begin{tabular}{|c|l|}
\hline Decibeles & \multicolumn{1}{|c|}{ Efecto } \\
\hline 30 & $\begin{array}{l}\text { Dificultad en conciliar el sueño. } \\
\text { Pérdida de calidad del sueño. }\end{array}$ \\
\hline 40 & Dificultad en la comunicación verbal. \\
\hline 45 & Probable interrupción del sueño. \\
\hline 50 & Malestar diurno. \\
\hline 55 & Malestar diurno fuerte. \\
\hline 65 & $\begin{array}{l}\text { Comunicación verbal } \\
\text { extremadamente difícil. }\end{array}$ \\
\hline 75 & Pérdida de oído a largo plazo. \\
\hline $110-140$ & Pérdida de oído a corto plazo. \\
\hline
\end{tabular}

Fuente: Morales ${ }^{[9]}$.

En particular en el ámbito escolar, los altos niveles de ruido, ya sea continuo o intermitente, obligan a que el maestro eleve su nivel de voz durante la jornada escolar, o incluso se deben volver a explicar los temas vistos, debido a la falta de comprensión por el ruido o, en el peor de los escenarios, el docente puede experimentar daños temporales o incluso permanentes en sus cuerdas vocales, todo esto impactando en la eficiencia de las actividades académicas ${ }^{[10]}$.

En México, la Secretaría del Trabajo y Previsión Social (STPS) cuenta con la norma NOM-011-STPS-2001, que se encarga de establecer las condiciones de seguridad e higiene en lugares donde se genere ruido que, por sus características, niveles y tiempos de acción, sea capaz de afectar la salud de los trabajadores. Esta norma se encuentra apoyada en otras ya existentes, por ejemplo, en una relativa al equipo de protección personal para los trabajadores en los centros de trabajo ${ }^{[11]}$.

La importancia de llevar a cabo evaluaciones del nivel de ruido presente en los ámbitos escolares y laborales se debe a que la exposición prolongada a ruidos elevados da origen al desarrollo de cansancio excesivo, así como a la disminución de la productividad. Se estima que, en el caso del trabajo intelectual, la capacidad laboral disminuye un $60 \%$ a causa de los altos niveles de ruido, mientras que en los trabajos donde predomina la actividad física, esta capacidad disminuye un $30 \%$, impactando en la fabricación de productos defectuosos $y$ accidentes laborales ${ }^{[12]}$.

Existe una necesidad por realizar proyectos orientados a la evaluación del ambiente escolar, en lo que a ruido respecta, dado que es relevante contar con lineamientos y medidas precisas para atender este problema de contaminación ${ }^{[13]}$. Este estudio de caso forma parte de la primera fase de evaluación del ambiente sonoro en la institución académica, específicamente aquellas aulas que la unidad administrativa tiene identificadas como lugares con necesidades de investigación. Se carece de estadísticas o número de quejas acerca de las mismas, sin embargo, por indicaciones de la misma institución y debido a su inquietud por solucionar esta problemática es que se decidió llevar a cabo este trabajo.

\section{METODOLOGÍA}

\section{A. Diseño de la investigación}

El diseño del presente proyecto es de tipo descriptivo, cuantitativo, no experimental y transversal. Se considera como una investigación descriptiva debido a que solo se representa y detalla la situación actual del área de estudio, a través de una medición e identificación del nivel de ruido generado en las aulas seleccionadas, para proporcionar una descripción de esta condición. 
Además es no experimental, debido a que se realiza sin manipular deliberadamente las variables y se considera también de tipo longitudinal ya que los muestreos se realizan en dos partes: en la primera son realizados con el aula vacía y en la segunda con el aula en uso (en clase), con el objeto de realizar las comparaciones pertinentes ${ }^{[14]}$.

\section{B. Materiales}

Los materiales utilizados para la medición descrita en este proyecto fueron un sonómetro marca Smart Sensor previamente calibrado, mismo que fue utilizado para el levantamiento de los datos correspondientes al nivel de ruido en las aulas; un tripié para situar el sonómetro en los puntos seleccionados y de esta forma ubicar el equipo a la altura que marca la norma correspondiente -distancia medida con un flexómetro-; y una hoja de registro, la cual fue diseñada con la finalidad de registrar las lecturas tomadas a lo largo de los muestreos. Esta contiene información tal como aula, límite de observación, fecha y tiempo en el que se realizó el levantamiento de datos e información del instrumento de medida, el tipo y límites de decibeles, así como las lecturas obtenidas a lo largo del estudio, las cuales fueron grabadas con un teléfono celular para posteriormente ser exportadas a la hoja de registro antes mencionada.

\section{Método}

Primero se identificaron las aulas a evaluar a través del uso de un layout de las instalaciones de estudio, las cuales tienen en común la falta de falso plafón. Este edificio está diseñado estilo industria, a diferencia del resto de los edificios en los que se cuenta con el falso plafón y no se han manifestado quejas acerca del ruido percibido. Se revisaron horarios de clase y cuando se encontraban libres, una vez asignadas las horas en que se realizarían los estudios en las distintas aulas, como primera fase se procedió a realizar el estudio en los salones que se encontraban desocupados. La toma de datos fue realizada de las 10:00 a las 14:00 horas.

Enseguida se instaló el sonómetro sobre un tripié a una altura aproximada de 1.5 metros, de acuerdo con lo establecido por la norma NOM-011-STPS-2011, la misma que indica que las mediciones deben ser realizadas en un periodo de exposición de 5 minutos o recabar 50 lecturas como mínimo. Con un total de cuatro puntos por prueba en cada aula, a una distancia aproximada de 3 metros de separación con la pared, esto para dar cumplimento con la norma mencionada.

Ubicando el sonómetro como se observa en la Figura 1 , se procedió a obtener las lecturas arrojadas por el instrumento, que fueron videograbadas a través de un teléfono celular, para posteriormente ser apuntadas en el formato diseñado para el registro de niveles en prueba de ruido. En la figura antes mencionada se pueden ver algunas de las pruebas realizadas en salones desocupados.

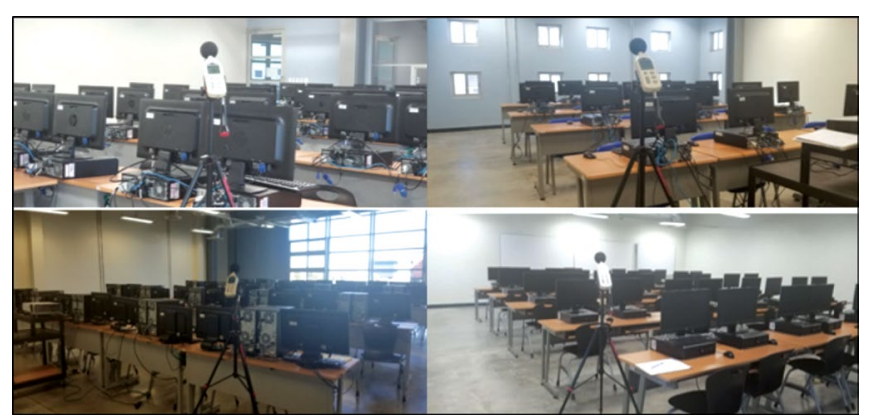

Figura 1. Estudio de ruido en aulas vacías.

Para la segunda etapa se solicitó la autorización al docente a cargo de la clase para ingresar al salón, explicándole previamente en qué consistía la prueba y el objetivo con el que sería realizado el estudio, así como indicándole que la clase debía continuar su curso de forma normal como siempre la lleva a cabo. Después de tener la anuencia para entrar al lugar, se tomaron los datos correspondientes con la clase en curso (Figura 2). Cabe mencionar que al realizar la toma de datos en los salones con clase, había 30 personas en cada una, ya que es la capacidad máxima de dichas aulas.

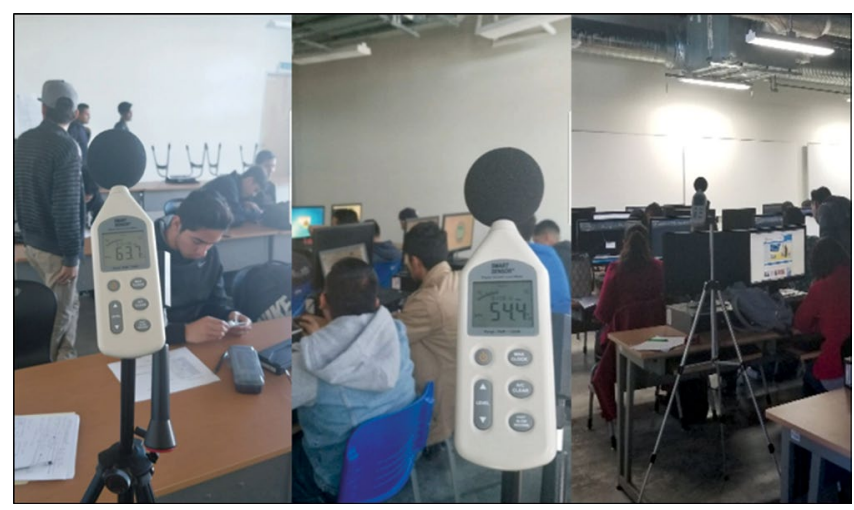

Figura 2. Estudio de ruido en aulas en uso.

Se procedió a registrar los datos en el software Minitab para obtener los estadísticos descriptivos y realizar el 
análisis correspondiente. Finalmente se compararon los decibeles obtenidos con lo establecido por la NOM011-STPS-2011, para determinar si las instalaciones cumplen con los niveles establecidos por la misma.

\section{RESULTADOS Y DISCUSIÓN}

\section{A. Resultados del estudio de ruido}

En la Tabla 2 se pueden observar los resultados de los estadísticos descriptivos de los niveles de ruido en aulas desocupadas. Los datos mostrados en la tabla corresponden a la media que indica el promedio de las lecturas, la desviación estándar muestra qué tan dispersos se encuentran los datos respecto a la media ya proporcionada, la moda detalla cuál es el valor con mayor presencia a lo largo del estudio, mientras que los niveles mínimos y máximos, como su nombre lo dice, representan el valor mayor y menor de la muestra obtenida y, por último, el rango de las mediciones proporciona información acerca de la diferencia entre el valor más elevado y el mínimo obtenido durante el estudio.

TABLA 2.

Estadísticos Descriptivos de los Niveles de Ruido eN Aulas Desocupadas

\begin{tabular}{|l|l|c|c|c|c|c|}
\hline Aula & Punto & $\overline{\mathbf{x}}$ & $\sigma$ & Mín & Máx & RANGo \\
\hline 1 & $\mathrm{~A}$ & 40.996 & 2.748 & 38.8 & 52.2 & 13.4 \\
\cline { 2 - 7 } & $\mathrm{B}$ & 40.128 & 0.876 & 38.9 & 44.3 & 5.4 \\
\cline { 2 - 7 } & $\mathrm{C}$ & 40.366 & 1.132 & 38.7 & 43.8 & 5.1 \\
\cline { 2 - 7 } & $\mathrm{D}$ & 42.306 & 1.508 & 40.1 & 46 & 5.9 \\
\hline \multirow{4}{*}{2} & $\mathrm{~A}$ & 39.286 & 0.833 & 38.9 & 38.6 & 43.1 \\
\cline { 2 - 7 } & $\mathrm{B}$ & 39.802 & 0.724 & 38.7 & 41.5 & 2.8 \\
\cline { 2 - 7 } & $\mathrm{C}$ & 39.736 & 1.235 & 38.5 & 45.5 & 7 \\
\cline { 2 - 7 } & $\mathrm{D}$ & 39.214 & 0.662 & 38.3 & 41.4 & 3.1 \\
\hline 3 & $\mathrm{~A}$ & 43.688 & 1.47 & 41.4 & 47.1 & 5.7 \\
\cline { 2 - 7 } & $\mathrm{B}$ & 41.414 & 1.78 & 39.8 & 47.6 & 7.8 \\
\cline { 2 - 7 } & $\mathrm{C}$ & 40.886 & 1.046 & 39 & 43.8 & 4.8 \\
\cline { 2 - 7 } & $\mathrm{D}$ & 40.798 & 0.678 & 39.8 & 43 & 3.2 \\
\hline 4 & $\mathrm{~A}$ & 41 & 1.283 & 39.5 & 44.5 & 5 \\
\cline { 2 - 7 } & $\mathrm{B}$ & 42.32 & 1.627 & 40.3 & 47.7 & 7.4 \\
\cline { 2 - 7 } & $\mathrm{C}$ & 42.712 & 1.621 & 41 & 47.5 & 6.5 \\
\cline { 2 - 7 } & $\mathrm{D}$ & 39.027 & 5.069 & 36.96 & 40.8 & 1.8 \\
\hline
\end{tabular}

Bajo el mismo entendido se realizó el análisis de los estadísticos descriptivos, pero esta vez con clase en curso, lo que arrojó los siguientes datos registrados en la Tabla 3, en la que se observa específicamente que en el aula 4, en el punto $\mathrm{D}$ se registró como valor máximo $74.4 \mathrm{dBA}$, seguido por el marcado con la letra $\mathrm{C}$ de la misma con $73.5 \mathrm{dBA}$ y a partir de ahí oscila entre 72.5 y $51.8 \mathrm{dBA}$.

TABLA 3.

Estadísticos Descriptivos de los Niveles de Ruido en Aulas Ocupadas

\begin{tabular}{|c|c|c|c|c|c|c|}
\hline Aula & Punto & $\overline{\mathrm{x}}$ & $\sigma$ & Mín & MÁx & RANGO \\
\hline \multirow[t]{4}{*}{1} & A & 64.364 & 4.263 & 55.4 & 72.5 & 17.1 \\
\hline & B & 60.81 & 3.409 & 54.6 & 70.6 & 16 \\
\hline & $\mathrm{C}$ & 57.512 & 6.471 & 44.8 & 68.8 & 24 \\
\hline & $\mathrm{D}$ & 57.076 & 4.77 & 48.7 & 66.4 & 17.7 \\
\hline \multirow[t]{4}{*}{2} & A & 56.196 & 3.559 & 48.1 & 64.6 & 16.5 \\
\hline & $\mathrm{B}$ & 61.012 & 4.469 & 49.2 & 72.4 & 23.2 \\
\hline & $\mathrm{C}$ & 52.308 & 4.685 & 45.1 & 66.6 & 21.5 \\
\hline & $\mathrm{D}$ & 53.23 & 4.817 & 44.4 & 66.3 & 21.9 \\
\hline \multirow[t]{4}{*}{3} & $\mathrm{~A}$ & 47.11 & 5.666 & 41.7 & 69.8 & 28.1 \\
\hline & $\mathrm{B}$ & 46.446 & 3.642 & 40.4 & 63.3 & 22.9 \\
\hline & C & 44.474 & 2.726 & 40.6 & 51.8 & 11.2 \\
\hline & $\mathrm{D}$ & 48.008 & 2.905 & 44.3 & 54.7 & 10.4 \\
\hline \multirow[t]{4}{*}{4} & $\mathrm{~A}$ & 61.438 & 5.052 & 48.5 & 72.2 & 23.7 \\
\hline & $\mathrm{B}$ & 49.238 & 4.861 & 41 & 60.6 & 19.6 \\
\hline & $\mathrm{C}$ & 67.692 & 3.088 & 59.2 & 73.5 & 14.3 \\
\hline & $\mathrm{D}$ & 68.108 & 2.927 & 61.8 & 74.4 & 12.6 \\
\hline
\end{tabular}

\section{B. Comparación de nivel de ruido}

En la Figura 3 se muestra la comparación entre los niveles de ruido promedio encontrados en cada una de las aulas evaluadas. Se puede observar que los niveles de ruido mientras está la clase en curso son más elevado que con las aulas vacías, sin embargo, ninguno de estos valores excede los $96 \mathrm{~dB}$ marcados por la norma.

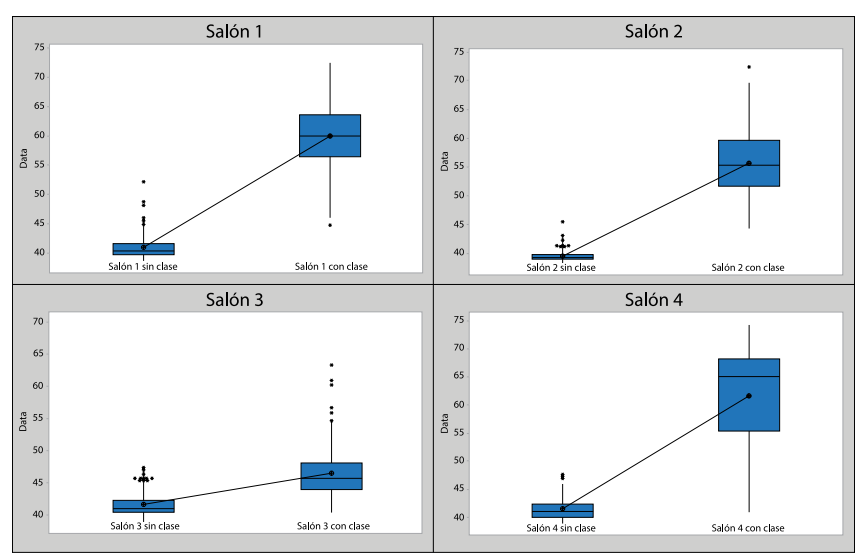

Figura 3. Comparación de decibeles en las cuatro aulas desocupadas y en uso. 


\section{CONCLUSIONES}

El principal objetivo del proyecto fue determinar los niveles de ruido en los salones de clase de una institución educativa, para conocer si se cumple con lo establecido por la NOM-011-STPS-2001, misma que indica niveles de exposición recomendados con respecto a un tiempo máximo permitido. Esto se logró a través del estudio de ruido que fue realizado en las cuatro aulas anteriormente indicadas.

Los niveles de ruido encontrados en las aulas vacías oscilaron entre 39.509 y $41.696 \mathrm{dBA}$, siendo el aula 2 la que obtuvo el promedio de media más bajo con 39.509 $\mathrm{dBA}$, mientras que el más elevado fue registrado en el salón 3 con $41.696 \mathrm{dBA}$. Por su parte, estas mismas aulas con clases en curso tuvieron una variación más alta, debido a la interacción típica de las actividades escolares. En estas aulas se contó con la presencia de 31 personas (30 estudiantes y 1 docente) que es la capacidad máxima de ellas. Se obtuvieron resultados de 46.509 dBA en el aula 3 y 61.619 dBA en el salón 4 .

Con respecto a la hipótesis planteada, que establecía como $\mathrm{H} 0$ que el nivel de ruido en las aulas bajo estudio se encontraba dentro de los límites establecidos por la NOM-011-STPS-2001, esta hipótesis no fue rechazada debido a que ninguna de las lecturas registradas en el estudio llegaron a superar los $96 \mathrm{dBA}$, que son los establecidos como nivel de exposición recomendado para un lapso máximo de exposición de dos horas, en virtud de que la duración habitual de las clases es de este tiempo. Por lo anterior, se concluye que las aulas evaluadas cumplen con los niveles de ruido establecidos por la norma antes mencionada.

A través de la evaluación que se realizó en dichas áreas, se pudieron detectar las zonas que contaban con mayor presencia de ruido, las cuales fueron el aula 1, con una media de $59.941 \mathrm{dBA}$, y el aula 4, con $61.619 \mathrm{dBA}$. A pesar de estos registros no se superó el permitido por la norma aplicada para este proyecto.

Es importante resaltar que las pruebas no fueron realizadas en un mismo periodo debido a que cada aula cuenta con horarios distintos. Se observaron diferencias notorias entre los salones con clase, dado que los alumnos presentan menor entusiasmo en las primeras horas de la mañana y por ello la emisión de ruido disminuía, como fue el caso de la prueba en el salón 3, que fue realizada en la clase correspondiente a las 08:00 horas y sus lecturas no se elevaron significativamente con respecto a las adquiridas en la prueba con lugar vacío, a diferencia de la muestra adquirida del salón 4 que fue tomada a las 11:00 horas y su variación fue más marcada con respecto al análisis en solitario.

Otro de los factores importantes es que el sistema de ventilación en dichas áreas se encontraba apagado debido a que fue en el mes de marzo cuando se realizaron los estudios, por lo cual es recomendable que las pruebas se hagan de manera periódica en distintas épocas del año cuando el sistema de refrigeración y calefacción se encuentre funcionando, con el fin de determinar si con este cambio en las condiciones del aula se presenta una elevación en el nivel de ruido y revalorar si se sigue dando cumplimiento a la norma.

También se sugiere realizar una comparación con las aulas del resto de los edificios en esta institución que cuentan con falso plafón en el techo, para determinar el efecto que tiene el diseño de las aulas sobre la presencia de ruido.

Se recomienda además diseñar un formato para registrar quejas respecto a esta problemática, con el objetivo de conocer en qué meses y horarios se presenta mayor molestia por parte de los docentes y en virtud de esto planificar la realización de los estudios con la finalidad de evaluar si en estos puntos se incumple con la norma y realizar acciones de mejora para la reducción si es necesario.

En caso de que las pruebas realizadas con las condiciones ya mencionadas resultaran estar dentro de los parámetros establecidos por la norma, se recomienda proceder a realizar un análisis del diseño interior de las aulas, en virtud de que la construcción física de los edificios no considera el aspecto acústico que se requiere para los espacios de aprendizaje mediante la utilización de materiales aislante.

El transmitir conocimiento de forma oral es un método comúnmente utilizado, el cual puede verse afectado por la presencia de eco y ruido en el aula, mismos que pueden ser potenciados en espacios con grandes volúmenes y mínima capacidad de absorción sonora, generando que el ruido se amplíe, así como por cuestiones como la capacidad de las paredes, aberturas y tabiques para impedir el paso del sonido exterior hacia el inte- 
rior del salón y viceversa. La mejora en la calidad de los espacios escolares debe ser primordial para el proceso enseñanza-aprendizaje debido al tiempo que los alumnos pasan en las aulas.

Esta situación genera que los alumnos, en consecuencia, no escuchen la participación de sus compañeros, pierdan concentración, genere fatiga y tengan que realizar un esfuerzo superior para entender el mensaje producido, provocando con esto que se pierda la continuidad y por tanto la calidad de la misma. Las aulas deben ser un espacio con ciertas características acústicas que permitan facilitar la transmisión de los mensajes que ahí se presentan.

\section{REFERENCIAS}

[1] STPS, Norma Oficial Mexicana NOM-011-SPTS-2001, Condiciones de seguridad e higiene en los centros de trabajo donde se genere ruido, 2002 [en línea]. Disponible en: http://www.cucba.udg.mx/sites/default/files/proteccioncivil/normatividad/Nom-011.pdf

[2] L. M. Chaux-Álvarez y B. Acevedo-Buitrago, "Evaluación de ruido ambiental en alrededores a centros médicos de la localidad Barrios Unidos, Bogotá", Revista Científica, vol. 2, no. 35, pp. 234-246, mayo-agosto2019.

[3] Y. Rodríguez y W. Baldeón, "Evaluación del ruido y el confort acústico en la Biblioteca Agrícola Nacional. Lima, Perú", Medicina y Seguridad del Trabajo, vol. 64, no. 250, pp. 17-32, ene./mar. 2018.

[4] B. Zamorano, F. Peña, V. Parra, Y. Velázquez y J. I. Vargas, "Contaminación por ruido en el centro histórico de Matamoros", Acta Universitaria, vol. 25, no. 5, pp. 20-27, sep./oct. 2015.

[5] E. A. C. Hermosillo y M. E. Escalante, "Influencia del ruido", Ciudad Juárez: Universidad Autónoma de Chihuahua, 1985.
[6] M. R. Párraga y T. García, "El Ruido y el Diseño de un Ambiente Acústico", Industrial Data, vol. 8, no. 2, pp. 83-85, 2005.

[7] P. Maijala, Z. Shuyang, T. Heittola y T. Virtanen, "Environmental noise monitoring using source classification in sensors", Applied Acoustics, vol. 129, pp. 258-267, enero 2018. https://doi.org/10.1016/j.apacoust.2017.08.006

[8] Y. González y Y. Fernández, "Efectos de la contaminación sónica sobre la salud de estudiantes y docentes, en centros escolares", Revista Cubana de Higiene y Epidemiología, vol. 52, pp. 402-410, 2014.

[9] C. Morales, "300 millones de personas sufren de contaminación acústica en el mundo: el ruido deja en silencio al planeta", Ciencia \& Trabajo, año 8, no. 20, pp. A45-A49, junio 2006.

[10] S. Beristain, "Ruido en Interior de Escuelas", Acústica 2004, pp. 1-6, 2004.

[11] STPS, Marco normativo de seguridad y salud en el trabajo, Secretaría del Trabajo y Previsión Social, 2012. [En línea]. Disponible en: http://asinom.stps.gob.mx:8145/ Centro/CentroMarcoNormativo.aspx

[12] M. C. Martínez, "Efectos del ruido por exposición laboral", Salud de los Trabajadores, vol. 3, no. 2, pp. 93-101, julio 1995.

[13] A. Figueroa, M. G. Orozco y N. E. Preciado, "Niveles de ruido y su relación con el aprendizaje y la percepción en escuelas primarias de Guadalajara, Jalisco, México," Ingeniería, vol. 16, no. 3, pp. 175-181, septiembre-diciembre 2012.

[14] R. Hernández, C. Fernández y P. Baptista, Metodología de la investigación. México: McGraw-Hill Interamericana, 2003. 